\title{
CORRESPONDENCE
}

\section{TO THE EDITOR}

\section{Stroke Training in Canadian Neurology Residency Training Programs}

\section{Re: tPA use for Stroke in the Registry of the Canadian Stroke Network. Can J Neurol Sci. 2005;32:433-9.}

Stroke patients now make up the majority of inpatient admissions to neurological wards and transient ischemic attack referrals, a growing number of outpatients. At our institution, for example, of the 911 inpatients admitted to the neurology clinical teaching unit in 2005, 611 were diagnosed with stroke. In the outpatient setting, 988 patients were assessed due to a possible transient ischemic attack. The Registry of the Canadian Stroke Network indicates that approximately $8.9 \%$ of patients presenting with an acute stroke in Canada are treated with thrombolysis. ${ }^{1}$ It is, therefore, important for neurology trainees to develop competency in the diagnosis and management of cerebrovascular disease.

There are many challenges faced by training programs in providing adequate stroke education to neurology trainees. How much time should be allocated to training in stroke? How much training should take place in the inpatient and outpatient setting? At what point during residency should this training take place? Should trainees play an active role in the acute stroke team and in the administration of thrombolysis? How will a trainee's competency and procedural skills be assessed?

There is a paucity of studies focusing on education provided to medical trainees in stroke. In a recent US survey, ${ }^{2} 27 \%$ of graduating neurology residents were not comfortable administering tPA independently and $20 \%$ had not personally treated patients with tPA. We were interested in determining the nature and amount of training in stroke provided to Canadian neurology residents. A questionnaire was developed and sent by email to all Canadian neurology residents in 2005 via the program directors of the 15 Canadian Adult Neurology Residency Training Programs. Despite a low response rate of $25 \%$ (34/136) limiting the interpretation of this study, we believe interesting and important information was still obtained. ${ }^{3}$

Of the 12 neurology training programs represented, six offered weekly educational stroke rounds. Half of the programs offered the opportunity for residents to undertake a local dedicated stroke elective. The duration of the stroke elective varied considerably from as short as a single month to several months in duration over the course of the residency program. A dedicated acute stroke service was present at 7 of 12 programs and residents participated in this service at all of these centers.

Twenty four of 34 residents $(71 \%)$ had personally administered thrombolysis. Fifty percent of these residents (12/24), however, had administered thrombolysis on only three or fewer occasions. As expected, experience with thrombolysis increased with each post graduate year, with a median of 0 (range $0-2$ ) cases in the first post graduate year to 7 (3-10) cases in the fifth post graduate year. There was one resident in the fourth post graduate year, however, who had never administered thrombolysis.
Despite the unevenness of dedicated training in stroke, $94 \%$ (32/34) of neurology residents were satisfied with their training in stroke. All trainees were comfortable or very comfortable with their knowledge of the modifiable risk factors for stroke and $91 \%$ (31/34) and 94\% (32/34) were comfortable or very comfortable with the indications for anticoagulation and carotid endarterectomy, respectively. In addition, $74 \%$ of residents who had administered thrombolysis, stated they would be comfortable administering thrombolysis independently in the future.

Some trainees may have acquired additional knowledge by attending the annual Canadian Stroke Consortium residents course (www.strokeconsortium.ca), which has taken place for the past six years. However, it is striking that despite the inadequacy of cerebrovascular training in most programs, the great majority of residents were satisfied with it. If they only knew what they do not know. Given the increasing prevalence of cerebrovascular diseases and the fact that stroke is both treatable and preventable, this situation calls for redress.

\section{Miguel Bussière, Vladimir Hachinski} London, Ontario, Canada

\section{REFERENCES}

1. Nadeau JO, Shi S, Fang J, Kapral MK, Richards JA, et al. tPA use for Stroke in the Registry of the Canadian Stroke Network. Can J Neurol Sci. 2005 32:433-9.

2. Cucchiara BL, Kasner SE. Graduating neurology residents' experience with IV tPA for acute stroke. Neurology. 2001;57:1729-30.

3. Bussière M, Hachinski V. Stroke education in Canadian neurology residency training programs. World Stroke Congress; 2005 Vancouver, B.C.

\section{To THE EdITOR}

\section{Re: Triphasic Waves versus Nonconvulsive Status Epilepticus: EEG Distinction. Can J Neurol Sci 2006;33:175-80.}

I wish to congratulate Dr Boulanger et al. ${ }^{1}$ for their recent article regarding the electroencephalographic distinction between triphasic waves (TWs) and generalized nonconvulsive status epilepticus (GNCSE). These authors approached reasonably an unresolved problem with obvious therapeutic implications. They concluded that some subtle morphological criteria seen on the electroencephalogram (EEG) and, particularly, the response to stimulation may be helpful in distinguishing TWs from GNCSE.

Nowadays, only a few researchers pay attention to establish simple and general principles for improving the EEG diagnosis in the evaluation of patients with altered mental state. Toxicmetabolic encephalopathy and GNCSE are common causes of delirium and, frequently, to obtain an accurate diagnosis depends on a precise and correct electroencephalographic interpretation. 